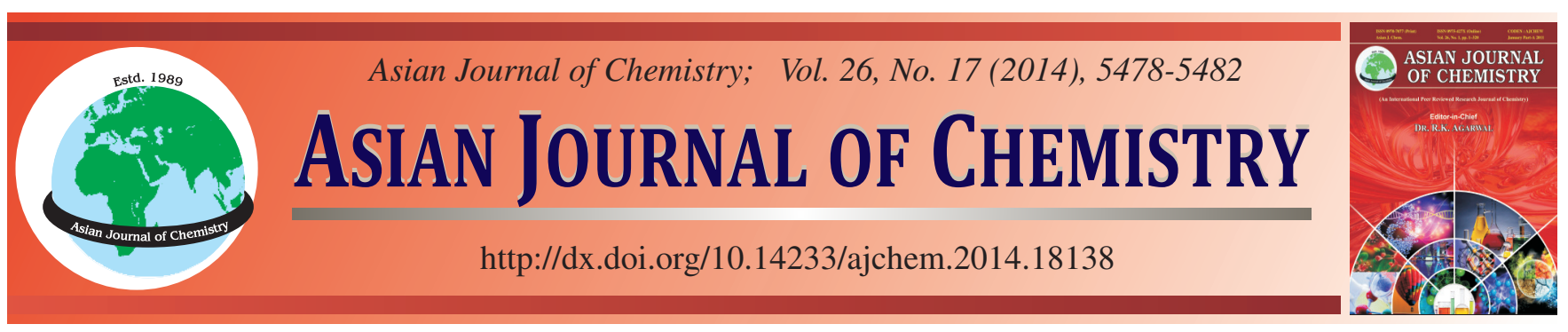

\title{
Simulation and Experimental Analysis of Influence of Inlet Flow on Heavy Hydrostatic Bearing Temperature Field $\dagger$
}

\author{
Yan Qin Zhang ${ }^{*}$, Wei Wei Li, Li Guo Fan, Rui Li and Jun Peng Shao
}

College of Mechanical \& Power Engineering, Harbin University of Science and Technology, Harbin 150080, P.R. China

*Corresponding author: Fax: +86 451 86390501; Tel: +86 13895736635; E-mail: yinsi1016@163.com

A multiple pad round guide was taken to solve the friction failure problem caused by the increase of the local temperature. The relationship between temperature and oil viscosity was systematically studied and an equation was established. Moreover, the fluid temperature field inside the hydrostatic bearing with a rotation speed of $6 \mathrm{rpm}$ was simulated at various flows and viscosities based on finite volume method. The influences of the inlet flow on the temperature of the clearance oil film were also studied. The results show that the shear flux plays an important role at low flux, while the stress difference flux on the clearance oil pad acts a vital part at high flux. In addition, the approximate high temperature area was searched out through numerical simulations of the oil film temperature field of the hydrostatic thrust bearing and then the effective temperature control was found out.

Keywords: Hydrostatic bearing, Temperature field, Various viscosity, Finite volume method.

ᄂ - - - - - - - - - - - - - - - - - - - - - - - - - - - - -

\section{INTRODUCTION}

The heavy hydrostatic bearing with multiple oil pad is the key part of the heavy CNC equipment due to its advantages of large bearing capacity, low friction coefficient and high working stability and reliability. Meantime, it is known that the performance of the bearing can affect the machining quality and working efficiency of the whole equipment directly. However, with the development of modern industry, the rotating machinery develops towards high speed, high accuracy and large power, resulting in the performance requirements of the bearing in all aspects become higher and higher. Hence, the research on heavy hydrostatic bearing is increasingly prompting to the width and the depth. When the hydrostatic bearing works under the pressure and heat conditions, local distortion would easily occur and the oil film would become thinner. Moreover, when the local oil film under boundary lubrication is in high speed heavy haul, dry friction will be brought out. The oil film will also break out because of the rapid increase at room temperature. Furthermore, the rotating velocity will be restricted by terminological failure. All those above will influence the machining accuracy and the work efficiency of the machine. In a word, deep theoretical research is the key point to improve the machining accuracy and work efficiency and to keep pace with the national mechanics environment developing towards high speed and high accuracy.
There are many researches on hydrostatic bearing, such as Niels and his co-workers established a mathematic model about 3-D thermal hydrodynamic lubrication based on Reynolds equation and studied the effects of oil cavity of tilting pad thrust bearings on capability of bearing. It suggests that shallow oil cavity has similar characteristics of step bearing ${ }^{1}$. Meantime, De Pellegrin and Dennis V took lots of studies on a sector-shaped pad in order to understand how recess size and shape affect the performance of a typical bearing ${ }^{2}$. Kumar and his coworkers analyzed a four-pocket capillary-compensated worn hydrostatic journal bearing system. Their studies indicate that bearing characteristics parameters are composed of maximum fluid-film pressure, minimum fluid-film thickness, lubricant flow rate and fluid film reaction ${ }^{3}$. The results of Okahata, et al. ${ }^{4}$ indicate that precision machine tools using hydrostatic water bearings are superior to those using hydrostatic oil bearings in terms of environmental protection, worker's safety and cost. As for hydrostatic water bearings, a constant-flow type is more suitable than a constant-pressure type. Nishitani et al. ${ }^{5}$ investigated the static and dynamic characteristics of water hydrostatic thrust bearings using porous material and compared it with conventional pocket hydrostatic bearings with a capillary restrictor. Hydrostatic porous bearings can be easily constructed because the porous material can develop into a viscous restrictor itself. Consequently, the water hydrostatic porous 
thrust bearings have higher maximum load capacity and slightly lower stiffness than water bearings with a capillary restrictor. Bakker and Ostayen ${ }^{6}$ determined the minimum dissipation for annular and circular recess thrust bearings, which was operated at a certain rotational speed by finding the optimized film thickness in the recess and gave an example for annular recess thrust bearings. In addition, many simulations were done by $\mathrm{Zhang}^{7}$ on turbine pump hydrostatic bearing using FLUENT software and then the inner flow state of hydrostatic bearing was discovered. The law load bearing capability and stiffness capability, which was affected by rotating velocity, filler oil pressure, excursion ratio, compensate diameter and oil film thickness, was also analyzed and the structure of bearing was optimized ${ }^{7}$. Fu et al. ${ }^{8}$ studied the three dimensional temperature field in a circular journal bearing by considering different factors, such as the variation of viscosity with temperature and pressure and the variation of density with viscosity and pressure. The temperature field in the oil film and bearing was obtained by solving the Reynold equation and the heat conduction equation, which was for a three dimension solid under the heat flux continuity conditions. The temperature distribution in the bearing with dynamic pressure oil film was obtained by using simulation method. The influence of the three dimensional temperature field on the practical lubrication performance of the bearing under a given load was analyzed. The results show that the variations of temperature in the oil of a hydrostatic bearing would change viscosity and the density of the oil ${ }^{9}$. Wang Ruichang ${ }^{10}$ did theorical calculation of the temperature field of the new thrust bearing on the basis of four-node using oparametric element and fluid mechanics finite element theory and then was verified by experimental results, which indicate a good coherence.

The influence of the viscosity and the rotation speed on the flow field and the temperature field of hydrostatic bearing was analyzed by 3D modeling software Pro/ENGINEER and CFD software FLUENT on the basis of the finite volume method $^{11-17}$. The deformation conditions, which was caused by the force and the heat of the hydrostatic bearing, were calculated through the finite element method. In the meantime, the cavity structure of the hydrostatic bearing was also optimized. All above have offered the theory basis for stable running of the bearing in engineering practice.

Clearance fluid model and control equation: The 3D solid geometric model of the intermediate oil film in hydrostatic bearing is generated by the $3 \mathrm{D}$ CAD software $\mathrm{UG}$, as shown in Fig. 1.

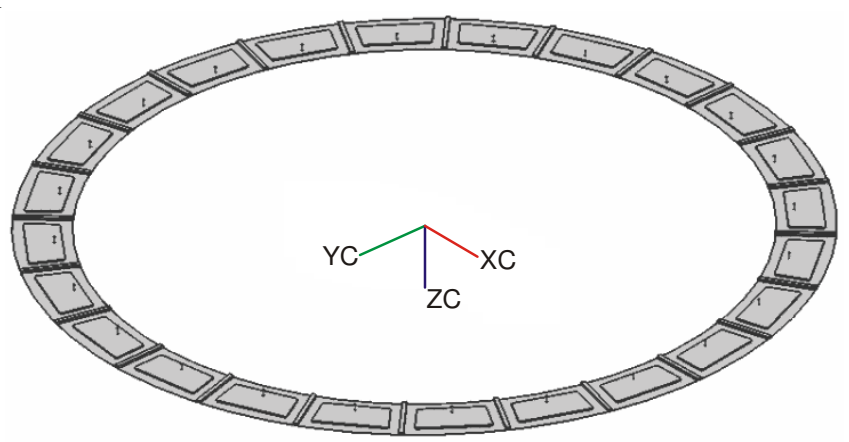

Fig. 1. Rectangular cavity clearance fluid model
Both the basic equations of fluid dynamics of the system and the control volume can be established. The conservation laws of mass, momentum and energy were applied into the relationships among fluid velocity, stress, density, temperature and other physical quantity obtained in fluid motion.

The basic equation is usually set on the control volume in the discussion of fluid dynamics. It has integral and differential forms ${ }^{18,19}$. The integral relationship of fluid physical quantities was obtained through quadrate the control volume and the control plane. The differential relationship of fluid physical quantities was obtained from any space point by mapping the equation of the micro-control element or the system directly. The general performance relation, such as the resultant force between objects and the main energy exchange, can be obtained through solving the basic equation in integral form. The flow details and the fluid physical quantities from any space point can be gained by solving the basic equation in differential form or the integral relationship of the micro-control element. The main differential equations are the mass conservation equation, the momentum conservation equation and the energy conservation equation.

The influence of centrifugal force should be taken into consideration due to the rotating motion droved by the rotating worktable. When the fluid rotates at a constant angular velocity of $\omega$ in the rotating frame, the new kinetic energy should take the influence of the Coriolis force and the centrifugal force into account. The relevant formulae are shown as follows:

$$
\begin{gathered}
S_{\text {cor }}=-2 \rho \omega \times U \\
S_{c f g}=-\rho \omega \times(\omega \times r) \\
S_{M, \text { rot }}=S_{\text {Cor }}+S_{c f g}
\end{gathered}
$$

where $\mathrm{S}_{\mathrm{Cor}}$ is the Coriolis force, $\mathrm{S}_{\mathrm{cfg}}$ is centrifugal force, $\mathrm{r}$ is the position vector of the micro-control element under rotational coordinate, $U$ is the velocity vector, $\omega$ is the rotational angle-velocity vector.

In the energy equation, the equation of total enthalpy is:

$$
\mathrm{I}=\mathrm{h}_{\text {stat }}+\frac{1}{2} \mathrm{U}^{2}-\frac{1}{2} \omega^{2} \mathrm{R}^{2}
$$

where $h_{\text {stat }}$ is the static enthalpy.

The mass conservation equation, the momentum conservation equation and the energy conservation equation can also be listed out by considering the centrifugal force obtained through the above equations.

The mass conservation equation is:

$$
\frac{\partial \rho}{\partial t}+\nabla \cdot(\rho U)=0
$$

where $\rho$ is the density, $t$ is the time. The density is a constant value because the research fluid is incompressible fluid, i.e., $\frac{\partial \rho}{\partial t}=0, \nabla$ is the divergence. That is:

$$
\nabla \cdot(\rho U)=\operatorname{div}(\rho U)=\frac{\partial(\rho u)}{\partial x}+\frac{\partial(\rho v)}{\partial y}+\frac{\partial(\rho w)}{\partial z}=0
$$

where u.v.w is the components in $\mathrm{x}, \mathrm{y}$ and $\mathrm{z}$ directions of velocity vector $\mathrm{U}$. 
The momentum conservation equation is:

$$
\begin{aligned}
\frac{\partial(\rho \mathrm{U})}{\partial \mathrm{t}}+\nabla\left(\rho \mathrm{U} \otimes \mathrm{U}_{\mathrm{abs}}\right)= & \nabla\left(-\mathrm{p} \delta+\mu\left(\nabla \mathrm{U}+(\nabla \mathrm{U})^{\mathrm{T}}\right)\right) \\
& -\rho \omega \times \mathrm{U}-\rho \omega \times(\omega \times \mathrm{r})
\end{aligned}
$$

where $\mathrm{p}$ is the stress on the fluid micro-element, $\mathrm{U}_{\mathrm{abs}}$ is the velocity vector on the absolute coordinate, is the viscosity, $\rho$ is the density.

The energy conservation equation is:

$$
\begin{aligned}
\frac{\partial\left(\rho \mathrm{h}_{\mathrm{tot}}\right)}{\partial \mathrm{t}}-\frac{\partial \mathrm{p}}{\partial \mathrm{t}}+\nabla \mathrm{g}( & \left.\rho \mathrm{Uh}_{\mathrm{tot}}\right)=\nabla \mathrm{g}(\lambda \nabla \mathrm{T}) \\
& +\nabla \mathrm{g}(\mathrm{Ug} \tau)+\mathrm{UgS}_{\mathrm{M}}+\mathrm{S}_{\mathrm{E}}
\end{aligned}
$$

where $\mathrm{h}_{\mathrm{tot}}$ is the total enthalpy, $\mathrm{T}$ is the temperature $\left({ }^{\circ} \mathrm{C}\right), \lambda$ is the fluid heat transfer coefficient of the fluid, $S_{M}$ is the momentum source, $S_{\mathrm{E}}$ is the inner heat source and part of the mechanical energy caused by the viscosity when it is converted into the heat energy.

In this article, it is necessary to point out that the energy conservation can be ignored because of the incompressible flow and without considering the heat transfer.

When the increase of temperature between inlet and outlet of lubricating oil is calculated, suppose that power consumption of liquid friction completely converts into heat and this heat could be completely absorbed by lubricating oil, according to energy conservation principle, so we can get maximum temperature rising between inlet and outlet of lubricating oil:

$$
\Delta \mathrm{t}=\mathrm{T}_{\text {in }}-\mathrm{T}_{\text {out }}=\frac{\mathrm{N}_{\mathrm{f}}}{\mathrm{c} \rho \Sigma \mathrm{Q}_{0}}
$$

where $T_{\text {in }}$ is oil pond temperature, $T_{\text {out }}$ is the temperature of oil which outflows from bearing gap, $\mathrm{c}$ is specific heat of lubricating oil, $\rho$ is density of lubricating oil, $\mathrm{N}_{\mathrm{f}}$ is friction power, $\mathrm{Q}_{0}$ is the total flow of a single oil pad.

However, it should be pointed out that $\Delta \mathrm{T}$ is directed as temperature difference value of oil out-flowing from oil edges and oil pond, but not absolute temperature rising of system and the latter lies on whole power consumption as well as heat emission condition.

Simplified model and mathematical model: GAMBIT software, a pre-processor provided by the FLUENT company, contains powerful geometric modeling capabilities and meshing tools. It could generate high-quality mesh model containing boundary layer, which will be directly used by FLUENT. Based on the idea of GAMBIT meshing, fluid model is cut into a number of regular geometric bodies in order to improve the mesh quality. Then model is divided into hexahedral meshes according to different body characteristics and meshed with cooper (unstructured mesh). Mesh quality of gap fluid will directly affect the accuracy of results. Fortunately, the number of first-grade grid will be over $90 \%$ through the mesh quality check.

Boundary conditions need to be set. "speed" will be selected as boundary condition for flow inlet and "pressure", i.e., atmospheric pressure, for flow outlet, . The top wall of gap fluid is defined as rotating wall, while the rest are defined as fixed wall. Two sides of gap fluid are set to periodic boundary conditions and it could ensure that only studying $1 / 24$ of gap fluid locates between static guide and rotating worktable, which could respond to flow state of overall the fluid. The film mesh of hydrostatic bearing is shown in Fig. 2 and the mesh check shown in Fig. 3. The setting of boundary type of a single oil pad is shown in Fig. 4.

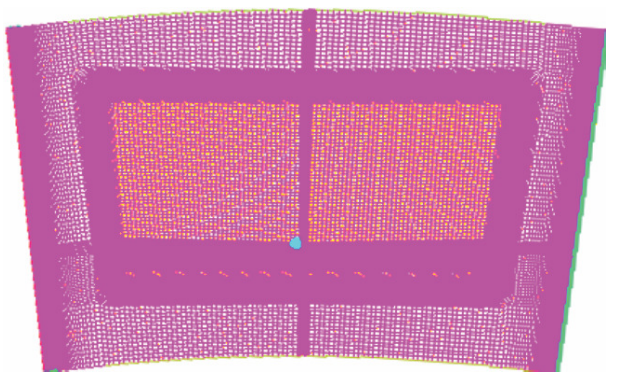

Fig. 2. Oil film mesh

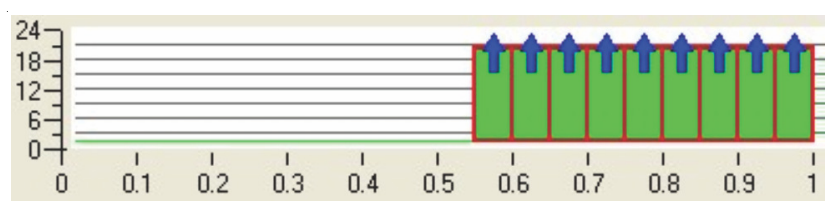

Fig. 3. Mesh check

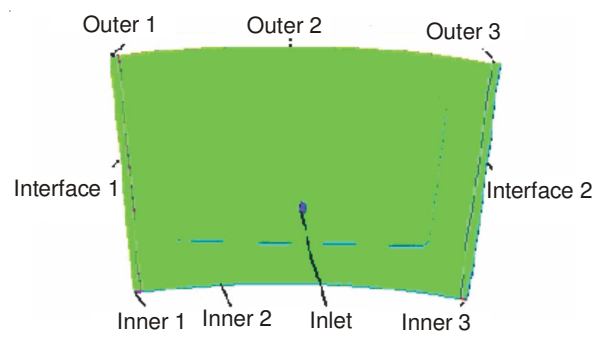

Fig. 4. Setting of boundary type

EXPERIMENTAL

The double-pillar vertical lathe DVT1000×50Q-NC is the major equipment of the test and the hydrostatic thrust bearing is one of the key components in the vertical lathe. The test device of hydrostatic thrust bearing system is shown in Fig. 5. In the experiment, a rotational part weighing 10 tons was used as load, working bench speed was changed in the way of poleless speed-adjusting and fuel supply system was regulated by variable displacement pump. The semiconductor thermometer with the measuring range $0-100^{\circ} \mathrm{C}$ and the resolution of $0.01{ }^{\circ} \mathrm{C}$ was used as the temperature indicator because of its high sensitivity and short response time. In the test of the oil film in heavy hydrostatic thrust bearing, temperature rising of the oil film was measured by the semiconductor thermometer.

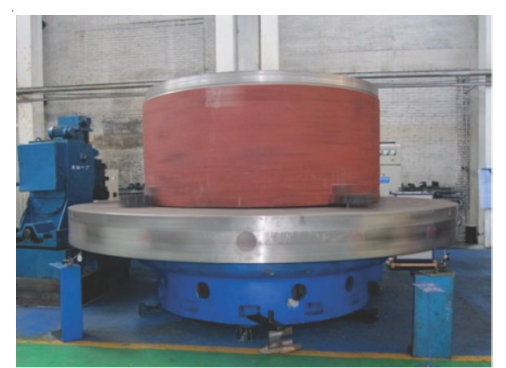

Fig. 5. Test device of hydrostatic thrust bearing system 


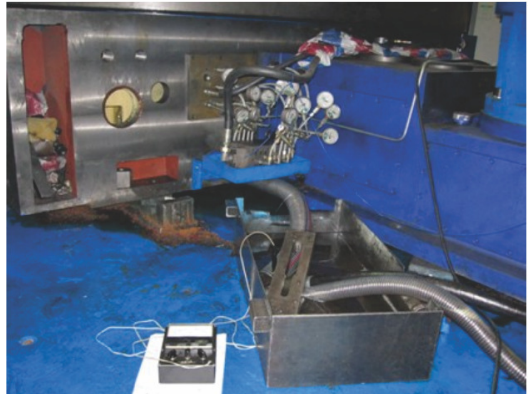

Fig. 6. Temperature rising experiment of hydrostatic bearing

The rising of temperature of the clearance oil film is measured based on the experimental apparatus above after the hydrostatic bearing reaching a steady state. The inlet flow rates are $6,12,18,24,30,36,42$ and $48 \mathrm{~L} / \mathrm{min}$, respectively, with the worktable rotation speed of $6 \mathrm{rpm}$. The inlet flow values are flow at different normal working levels of flow heavy machine with hydrostatic bearing. The data are shown in Table1 and the temperature rising curve of oil film obtained according to the experiment datum is shown as Fig. 7.

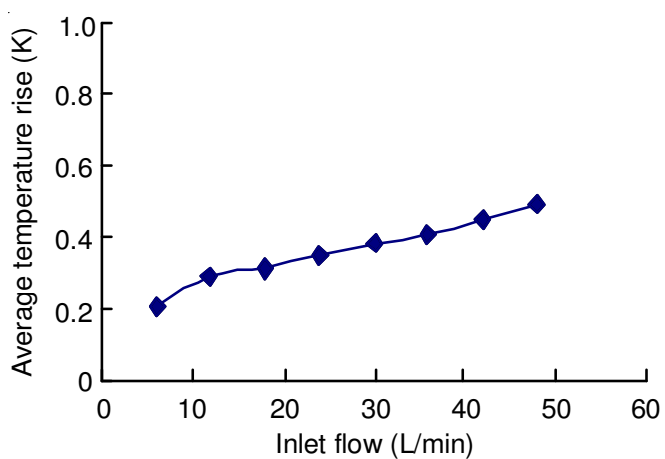

Fig. 7. Experimental relation chart of inlet flow and average temperature rising

It is clear from the experimental curve between inlet flow and average temperature rise, the average temperature rising value increases with the increase of inlet flow. It indicates that the average temperature rise speeds up gradually. The results show that the error between experiment and the simulation is within $\pm 3.5 \%$ and the error mainly comes from the loss of pipeline, the surface machining error, etc. So, it can be indicated that the temperature rising value of experimental results in flow variations is almost same as that in simulations. It also illustrates the credibility of simulation and the correctness of theoretical mathematical model.

\section{RESULTS AND DISCUSSION}

The working medium of the hydrostatic bearing is ISOVG 46 hydraulic oil. The main performances are as follows: the density $\rho=880 \mathrm{~kg} / \mathrm{m}^{3}$, the specific heat $\mathrm{C}=1884 \mathrm{~J} / \mathrm{kg} \mathrm{K}$, the kinematic viscosity $\mathrm{v}$ is $41.4-50.6 \mathrm{~mm}^{2} / \mathrm{s}$ at the fluid temperature of $40^{\circ} \mathrm{C}$, thermal conductivity $\lambda=0.132 \mathrm{w} / \mathrm{m} \mathrm{K}$, the relevant dynamic viscosity $\mu$ is $0.036432-0.044528 \mathrm{~Pa} \mathrm{~s}$. Considering that the worktable rotation speed in counterclockwise is constant in various viscosities, then the temperature field of the clearance oil film in hydrostatic bearing can be simulated with the load of 10 tons and the worktable rotation speed at $6 \mathrm{r} / \mathrm{min}$, as the inlet flow rates are 6, 24, 30 and 48 $\mathrm{L} / \mathrm{min}$, respectively. The temperature distributions are shown as Figs. 8-11.

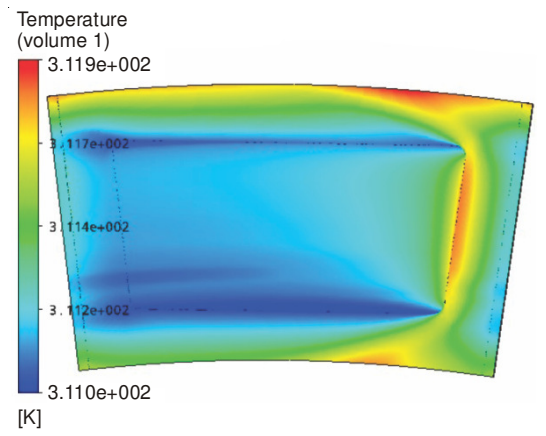

Fig. 8. Temperature field of $6 \mathrm{~L} / \mathrm{min}$ inlet flow

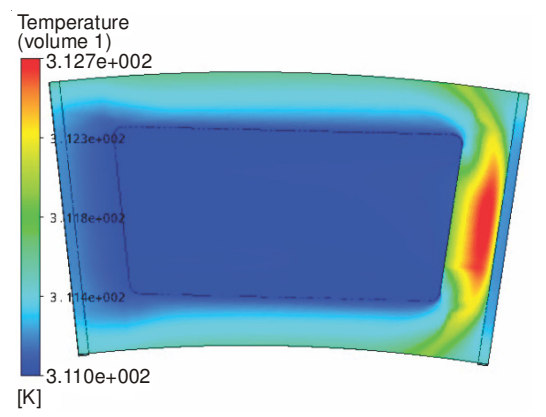

Fig. 9. Temperature field of $24 \mathrm{~L} / \mathrm{min}$ inlet flow

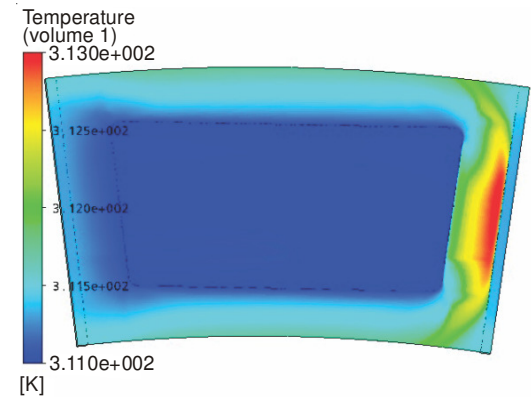

Fig. 10. Temperature field of $30 \mathrm{~L} / \mathrm{min}$ inlet

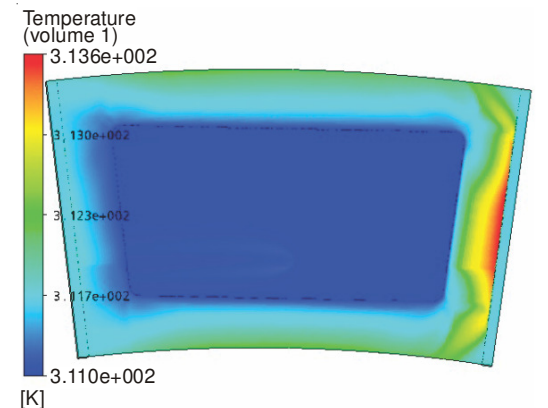

Fig. 11. Temperature field of $48 \mathrm{~L} / \mathrm{min}$ inlet flow

\begin{tabular}{lccccccccc}
\multicolumn{1}{c}{ TABLE-1 } \\
\begin{tabular}{ll} 
AVERAGE TEMPERATURE RISE IN DIFFERENT INLET FLOW \\
\hline Inlet flow rate $(\mathrm{L} / \mathrm{min})$
\end{tabular} & 6 & 12 & 18 & 24 & 30 & 36 & 42 & 48 \\
\hline Average temperature rise $(\mathrm{K})$ & 0.21 & 0.29 & 0.31 & 0.35 & 0.38 & 0.41 & 0.45 & 0.50 \\
\hline
\end{tabular}


TABLE-2

AVERAGE TEMPERATURE RISE IN DIFFERENT INLET FLOW

\begin{tabular}{|c|c|c|c|c|c|c|c|c|}
\hline Inlet flow rate $(\mathrm{L} / \mathrm{min})$ & 6 & 12 & 18 & 24 & 30 & 36 & 42 & 48 \\
\hline Inlet flow rate $(\mathrm{L} / \mathrm{min})$ & 0.28 & 0.318 & 0.342 & 0.354 & 0.393 & 0.434 & 0.478 & 0.523 \\
\hline
\end{tabular}

As seen from the above temperature simulations, distribution laws of temperature under different flow rates are almost the same. The highest temperature value is presented near the right oil edges and it decreases from right to left when working table is in anticlockwise rotation. The temperature distribution is asymmetry due to the flow value varies throughout the oil edges.

The relation chart of inlet flow and average temperature rising can be drawn out on the basis of Table-2 (Fig. 12).

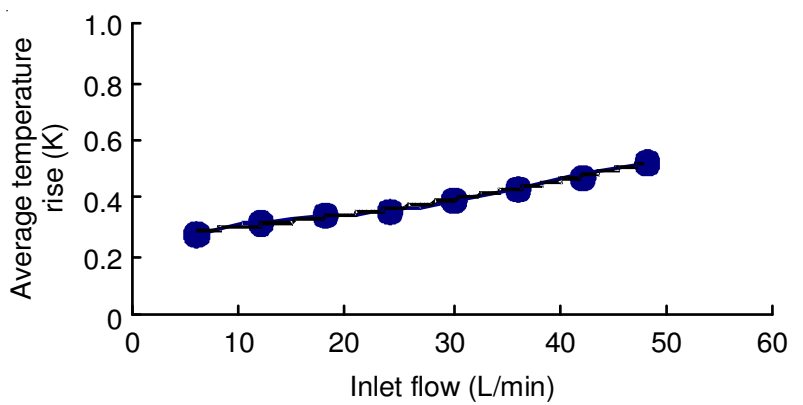

Fig. 12. Relation chart of inlet flow and average temperature rise

According to Fig. 8, the shear flux plays a vital role, so the temperature is in sequence of the temperature rising of the oil film at the verge of the oil pad, on the right of the oil pad, inside the oil pad, on the left of the oil pad from high to low. The stress difference flux acts an important part when the rotation speed is of $6 \mathrm{rpm}$ and the inlet flow rate is of 12-36 L/ $\min$. The temperature is in order of the temperature rising of the oil film on the right of the oil pad, at the verge of the oil pad, inside the oil pad, on the left of the oil pad from high to low. The average temperature rise increases with the increase of the inlet flow rate, but it has little effect on the temperature rising of the oil film in hydrostatic bearing.

\section{Conclusion}

The inlet flow rate greatly influences the temperature distribution conditions of a single oil pad in hydrostatic thrust bearing. The shear flux plays an important role at low flux and the high temperature area is on the right of the oil pad. The stress difference flux on the clearance oil pad acts a vital part at high flux and the high temperature area is on the right. The temperature rising increases slowly with the increase of inlet flow rate and the inlet flow rate has little effect on the temperature rising of the film in hydrostatic bearing. Divergence phenomena does not present in simulations. It indicates that the equation using finite volume method is stable. At the same time, the flow distribution of interstitial fluid on hydrostatic guide is in accordance with the practical distribution.

\section{ACKNOWLEDGEMENTS}

This material is based upon work Supported by the National Natural Science Funds for Young Scholar of China (No. 51005063), the Heilongjiang Postdoctoral Science-Research Foundation(LBH-Q12062) and Projects of the Special Fund on the Science and Technology Innovation People of Harbin, China (2013RFQXJ086).

\section{REFERENCES}

1. N. Heinrichson, I.F. Santos and A. Fuerst, J. Tribol., 129, 895 (2007).

2. D.V. De Pellegrin and D.J. Hargreaves, Tribol. Int., 51, 25 (2012).

3. E. Vijay Kumar, V.M. Phalle, S.C. Sharma and S.C. Jain, Analysis of a Worn Multirecess Hydroststatic Journal Bearing System', Proceedings of ASME 2009 International Design Engineering Technical Conferences \& Computers and Information in Engineering Conference, IDETC/CIE-2009, Paper No. DETC 2009-86787, San Diego, Californiia USA, August 30- Sept. 2 (2009).

4. O. Go and O. Shigeki, Numerical Study on Constant-Flow Hydrostatic Water Bearing for a Machine-Tool Table, 14th International Symposium on Advances in Abrasive Technology, 325, pp. 357-362 (2011).

5. Y. Nishitani, S. Yoshimoto and K. Somaya, Int. J. Automation Technol., 5, 773 (2011).

6. O.J. Bakker and R.A.J. van Ostayen, J. Tribol., 132, 1 (2010).

7. Y. Zhang, Dynamic Characteristic Analysis and Structure Optimization for the Hydrostatic Bearing of Turbo Pump, Harbin Institute of Technology, Harbin, pp. 17-54 (2007).

8. Y.L. Fu, X.Z. Ma and J. Zhu, Mechan. Sci. Technol. Aerospace Eng., 21, 711 (2002).

9. C.-M. Wang, Machnical Manage. Develop., 23, 101 (2008).

10. The Heavy Machnics, 6, 38 (2002).

11. J.P. Shao, C.- Dai, Y.- Zhang, X.- Yu, X.- Xu and Y.- Wang, J. Hydrodynam., 23, 676 (2011).

12. J.P. Shao, Y.Q. Zhang, Y.- Li, X.- Yu and H. Jiang, J. Central South Univ. Technol., 15(S2), 245 (2008).

13. J.P. Shao, Y.Q. Zhang and P.C. Li, Lubrication Eng., 32, 93 (2007).

14. Y.Q. Zhang and X.D. Yang, J. Hydrodynam., 25, 823 (2013).

15. Y.Q. Zhang, X.D. Yu, X.D. Yang, G.T. Sun, X.Y. Yu and Z.M. Shi, Key Eng. Mater. 450, 63 (2010).

16. X. Yu, X. Meng, H. Jiang, X. Lou, H. Xiang, J. Wang, T. Liu, C. Yang, X. Sun, W. Ji and D. Chen, Adv. Sci. Lett., 4, 2738 (2011).

17. X.-D. Yu, Key Eng. Mater, 419, 141 (2010).

18. P. Zhi-Cheng, Hydrostatic and Pneumostatic Technology, Heilongiiang People Publishing Company, Harbin, pp. 59-75 (1981).

19. W. Fu-Jun, Calculation Flow Mechanics Analyses FLUENT Software Principle and Applies, Tsing Hua University Press, Beijing, pp. 45-50 (2004). 